\title{
Low and Ultra-low Energy Scanning Electron Microscopy of 2D Transition Metal Dichalcogenides: Experiments and Simulations
}

Eliška Mikmeková ${ }^{1}$, Aleš Paták ${ }^{1}$, Ilona Müllerová ${ }^{1}$, Luděk Frank ${ }^{1}$, Benjamin Daniel ${ }^{1}$, Ivo Konvalina ${ }^{1}$, Tomáš Řiháček ${ }^{1}$, Martin Zouhar ${ }^{1}$, Anna Zaporozchenko ${ }^{2}$ and Michaël Lejeune ${ }^{3}$

1. Department of Electron Microscopy, Institute of Scientific Instruments CAS, v.v.i., Brno, Czech Republic.

2. Institute of Physics, University of Mainz, Mainz, Germany.

3. Laboratoire de Physique de la Matière Condensée, Université de Picardie Jules Verne, Amiens, France.

Two-dimensional (2D) materials have attracted huge interest since isolating of free-standing graphene. The reason is their interesting properties and promising potential applications [1]. Scanning electron microscopy with slow and very slow electrons offers an innovative tool enabling to study 2D materials in detail. In order to examine mutually overlapped flakes of two-dimensional crystals such as $2 \mathrm{D}$ transition metal dichalcogenides with an electron microscope we need to obtain a contrast contribution from a single layer of atoms. This task requires increasing the scattering rate of incident electrons by means of a drastic lowering of their energy to hundreds of eV or less.

Scanning Low Energy Electron Microscopy (SLEEM) was used to distinguish, or even count, TiSe $\mathrm{S}_{2}$ and $\mathrm{NbSe}_{2}$ layers on silicon substrate in the reflection mode. The samples were prepared by mechanical exfoliation (scotch tape method) and they were placed on the silicon wafer. While the low energy mode $100 \mathrm{eV} \leq \mathrm{E} \leq 5000 \mathrm{eV}$ is sufficient to distinguish the individual layers, the ultralow energies have a potential to count the layers from characteristic oscillations in the reflection mode. The position of minima in reflection spectra are in good agreement with a theoretical model based on the first principle Density Functional Theory (DFT).

As the most important advantage of SLEEM for study of 2D materials can be highlighted its very small interaction volume of impacted electrons. It means that the major part of the signal comes from the studied materials as presented in Figure 1. For illustration, the simulations of interaction volumes were provided by Monte Carlo simulations in software Casino [2].

Ultra-low energy electron reflectivity computations are performed using the Quantum Espresso suite for quantum simulation of materials [3]. The local density approximation with spin-orbit coupling, norm-conserving fully-relativistic pseudopotentials [4], and Perdew-Zunger exchange-correlation are used in the simulation. To add spin-orbit interaction we have modified Bloch wave matching algorithm from J. McClain thesis [5]. The images and DFT simulation of $\mathrm{NbSe}_{2}$ multilayers are presented in Figure 2. [6]

References:

[1] G R Bhimanapati et al, ACS Nano 9 (2015), p. 11509.

[2] D Drouin et al, Scanning 29 (2007), p. 92.

[3] P Giannozzi et al, Journal of Physics: Condensed Matter 21 (2009), p. 395502.

[4] A Dal Corso, Computational Materials Science 95 (2014), p. 337.

[5] J McClain, Doctoral dissertation, University of New Hampshire (2015).

[6] The authors acknowledge funding from the Technology Agency of the Czech Republic (Competence center Electron microscopy, no: TE01020118). 


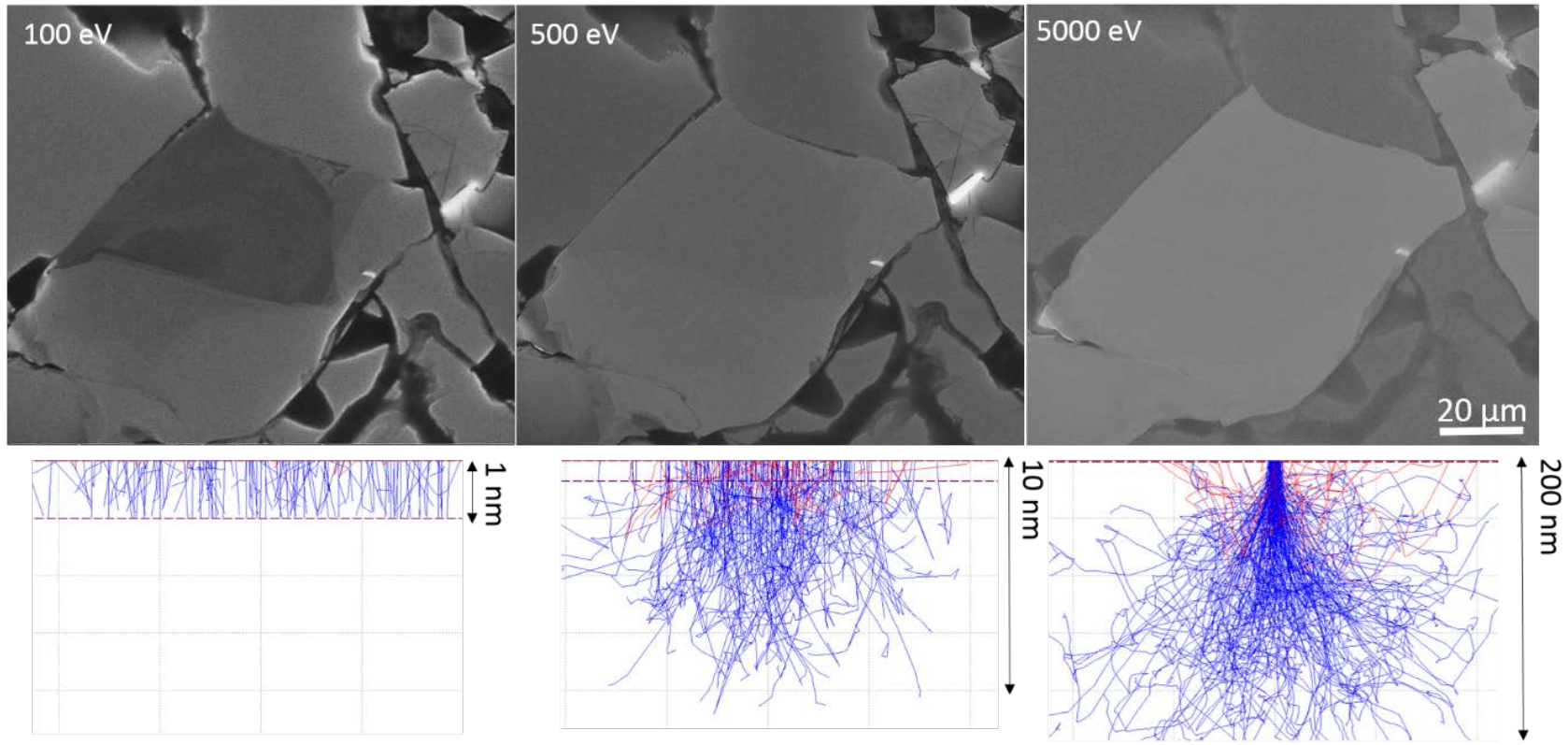

Figure 1. $\mathrm{TiSe}_{2}$ flakes observed at $100 \mathrm{eV}, 500 \mathrm{eV}$ and $5000 \mathrm{eV}$ by SLEEM and Monte Carlo simulations of interaction volumes (blue trajectories: forward scattered electrons, red trajectories: backscattered electrons).
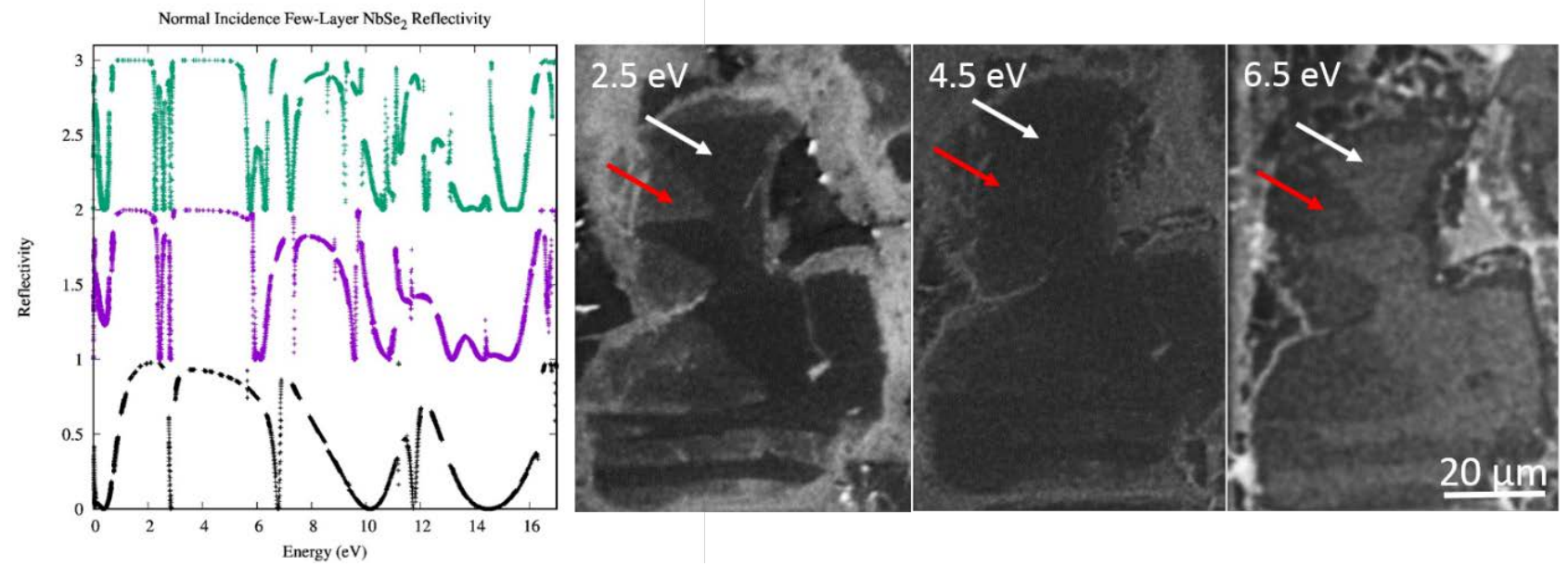

Figure 2. Simulated low energy electron reflectivity spectra of free-standing $2 \mathrm{H}-\mathrm{NbSe}_{2}$ for 1,2 and 3 layers from bottom to top, curves are shifted vertically in integers for clarity (left). The inversion of contrast was observed at landing energies $2.5 \mathrm{eV}, 4.5 \mathrm{eV}$ and $6.5 \mathrm{eV}$ (right). 